TRANSACTIONS OF THE

AMERICAN MATHEMATICAL SOCIETY

Volume 352, Number 2, Pages 655-677

S 0002-9947(99)02233-3

Article electronically published on September 9, 1999

\title{
CLOSED INCOMPRESSIBLE SURFACES IN KNOT COMPLEMENTS
}

\author{
ELIZABETH FINKELSTEIN AND YOAV MORIAH
}

\begin{abstract}
In this paper we show that given a knot or link $K$ in a $2 n$-plat projection with $n \geq 3$ and $m \geq 5$, where $m$ is the length of the plat, if the twist coefficients $a_{i, j}$ all satisfy $\left|a_{i, j}\right|>1$ then $S^{3}-N(K)$ has at least $2 n-4$ nonisotopic essential meridional planar surfaces. In particular if $K$ is a knot then $S^{3}-N(K)$ contains closed incompressible surfaces. In this case the closed surfaces remain incompressible after all surgeries except perhaps along a ray of surgery coefficients in $\mathbb{Z} \oplus \mathbb{Z}$.
\end{abstract}

\section{INTRODUCTION}

Incompressible surfaces play a major role in the study of 3-manifolds. In the case of manifolds with nonempty boundary, the existence of incompressible surfaces with boundary is a well-known result (see [He]). However, deciding whether a 3-manifold, closed or with boundary, contains a closed incompressible surface not parallel to the boundary turned out to be a hard problem.

An incompressible and boundary incompressible surface not parallel to the boundary will be called an essential surface. A surface in $S^{3}-N(K)$ with nonempty boundary will be called meridional if its boundary components are meridians for $K$.

Recall that all knots and links $K \subset S^{3}$ are known to have $2 n$-plat projections for some $n$ (see Definition 1.6 and [BZ]). Our main theorem is:

Theorem 1.1. Let $K \subset S^{3}$ be a knot or a link in a $2 n$-plat projection, with $n \geq 3$ and $m \geq 5$. If $\left|a_{i, j}\right|>1$, for each $i$ and $j$, then $S^{3}-N(K)$ contains at least $2 n-4$ nonisotopic essential meridional planar surfaces.

As immediate corollaries we obtain

Theorem 1.2. Let $K \subset S^{3}$ be a knot admitting a $2 n$-plat projection, with $n \geq 3$ and $m \geq 5$. If $\left|a_{i, j}\right|>1$, for each $i$ and $j$, then $S^{3}-N(K)$ contains a closed essential surface.

Proof. It follows from Theorem 2.0.3 of [CGLS] that if $K$ is a knot and if $S^{3}-N(K)$ contains an incompressible planar meridional surface, then $S^{3}-N(K)$ contains a closed essential surface (see also Theorem 6.1 of $[\mathrm{GR}]$ ).

Theorem 1.3. Let $K \subset S^{3}$ be a knot in a $2 n$-plat projection, $n \geq 3$ and $m \geq 5$. If $\left|a_{i, j}\right|>1$, for each $i$ and $j$, then the knot $K$ is a nontrivial knot.

Received by the editors May 23, 1996 and, in revised form, October 10, 1997.

1991 Mathematics Subject Classification. Primary 57M25, 57M99, 57N10.

(C)1999 American Mathematical Society 

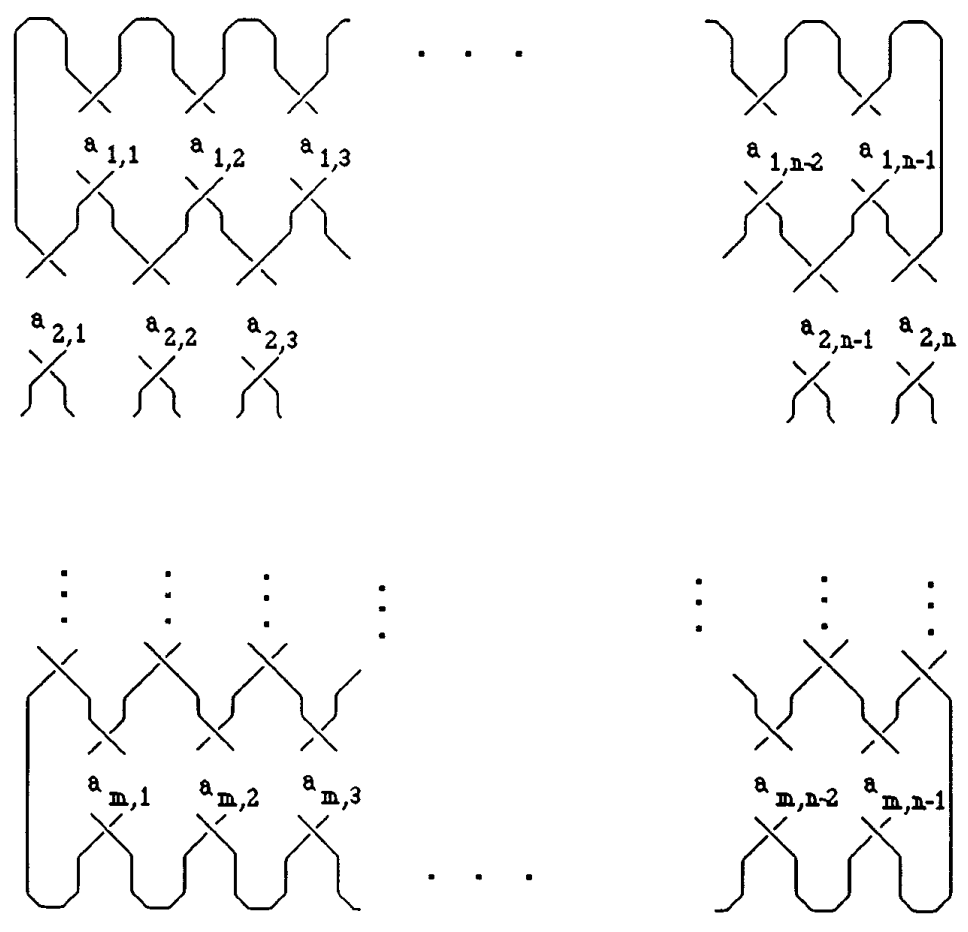

FiguRe 1

Remark 1.4. The condition that $\left|a_{i, j}\right|>1$ can be weakened; this is done in Lemma 5.1 of Section 5. However, the statement we obtain there is probably still not the best possible.

Remark 1.5. Theorem 1.2 is applicable to knots only since there are examples of links, for example the Boromean rings, which contain essential planar meridional surfaces but do not contain closed incompressible surfaces.

Definition 1.6. A knot/link $K \subset S^{3}$ has a $2 n$-plat projection if it can be projected onto a $2 n$-braid in some plane with "bridges" connecting the strings on the top and on the bottom, so that the number of rows of crossings, $m$, is odd, as indicated in Figure 1 (see also [BZ]). Each configuration of the form

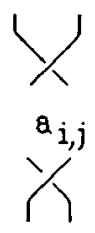

or

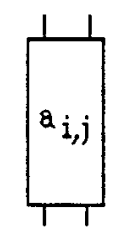


occurring in the $i$ th row and the $j$ th column, indicates $\left|a_{i, j}\right|$ crossings which are positive or negative depending on the sign of $a_{i, j}$, where $1 \leq i \leq m$ and $1 \leq j \leq n-1$ or $1 \leq j \leq n$, depending on whether the row is odd or even numbered respectively.

Remark 1.7. The fact that link complements contain essential surfaces is not that surprising since one can conceivably arrange the components of the link to be on either side of the surface in such a way that the surface is incompressible. However, the fact that essential surfaces are so abundant in knot complements is quite remarkable.

It follows from [Wu 1] that if the knot is not isotopic onto an incompressible surface, these surfaces will remain incompressible for all surgery coefficients in the surgery space, except perhaps for surgery coefficients on a single line. Let $\Delta(\gamma, \delta)$ denote the geometric intersection between geodesic curves on $\partial N(K)$ representing the surgery slopes $\gamma$ and $\delta$. We now have

Theorem 1.8. Let $K \subset S^{3}$ be a knot in a $2 n$-plat projection, $n \geq 3, m \geq 5$, with meridian $\mu$. If $\left|a_{i, j}\right|>1$, for each $i$ and $j$, and $K$ is not isotopic onto the essential surface, then all manifolds obtained by surgery, except surgery along slopes $\gamma$ such that $\Delta(\gamma, \mu) \leq 1$, are Haken.

Remark 1.9. It is conjectured by Lopez that every non-Haken 3-manifold contains a knot whose complement contains no closed incompressible surface. The Lopez conjecture has implications to some approaches toward solving the Poincare Conjecture. Theorem 1.2, which deals only with knots in $S^{3}$, explains why the Lopez conjecture is hard to prove.

The known results about closed incompressible surfaces have been of three types. The first type of results consist of various specific methods of constructing manifolds which contain essential surfaces and hence tend to be of very special nature (see for example $[\mathrm{Sw}])$. The second type assumes that essential surfaces exist in the 3manifold and then it is shown that these surfaces must have certain properties (see $[\mathrm{Sh}],[\mathrm{Me}])$. The third type of results are actual existence theorems and there are surprisingly few of them. The only families of knots or links whose complements are known to contain essential surfaces, beside obvious constructions like satellite knots, are fibered knots (see [Ly]), Star links, (which include Montesinos knot/links), (see $[\mathrm{Oe}]$ ), closed hyperbolic 3-braids (see [LP], [Fi]), Brunnian rings of at least four components, and homology boundary links (see [CL]).

\section{6-PLATS}

In this section we will discuss knots or links which have projections as 6-plats (see for example Figure 2). This will be a fundamental case in our argument.

Definition 2.1. An $n$-tangle, $n>1$, is a pair $(B, T)$ where $B$ is a 3 -ball and $T$ is a collection of $n$ disjoint arcs (strings) $t_{1}, \ldots, t_{n}$ properly embedded in $B$. A tangle will be called essential if the planar surface $\partial B-N(\partial T)$ is incompressible in $B-N(T)$.

Definition 2.2. Let $K$ be a knot or link in a 6-plat projection. A vertical 2sphere in $\left(S^{3}, K\right)$ is a 2 -sphere intersecting $N(K)$ in meridional curves separating $\left(S^{3}, K\right)$ into two tangles $\left(B_{1}, T_{1}\right),\left(B_{2}, T_{2}\right)$. Furthermore, the 2 -sphere intersects the projection plane of the plat in a unique simple closed curve. This curve intersects 


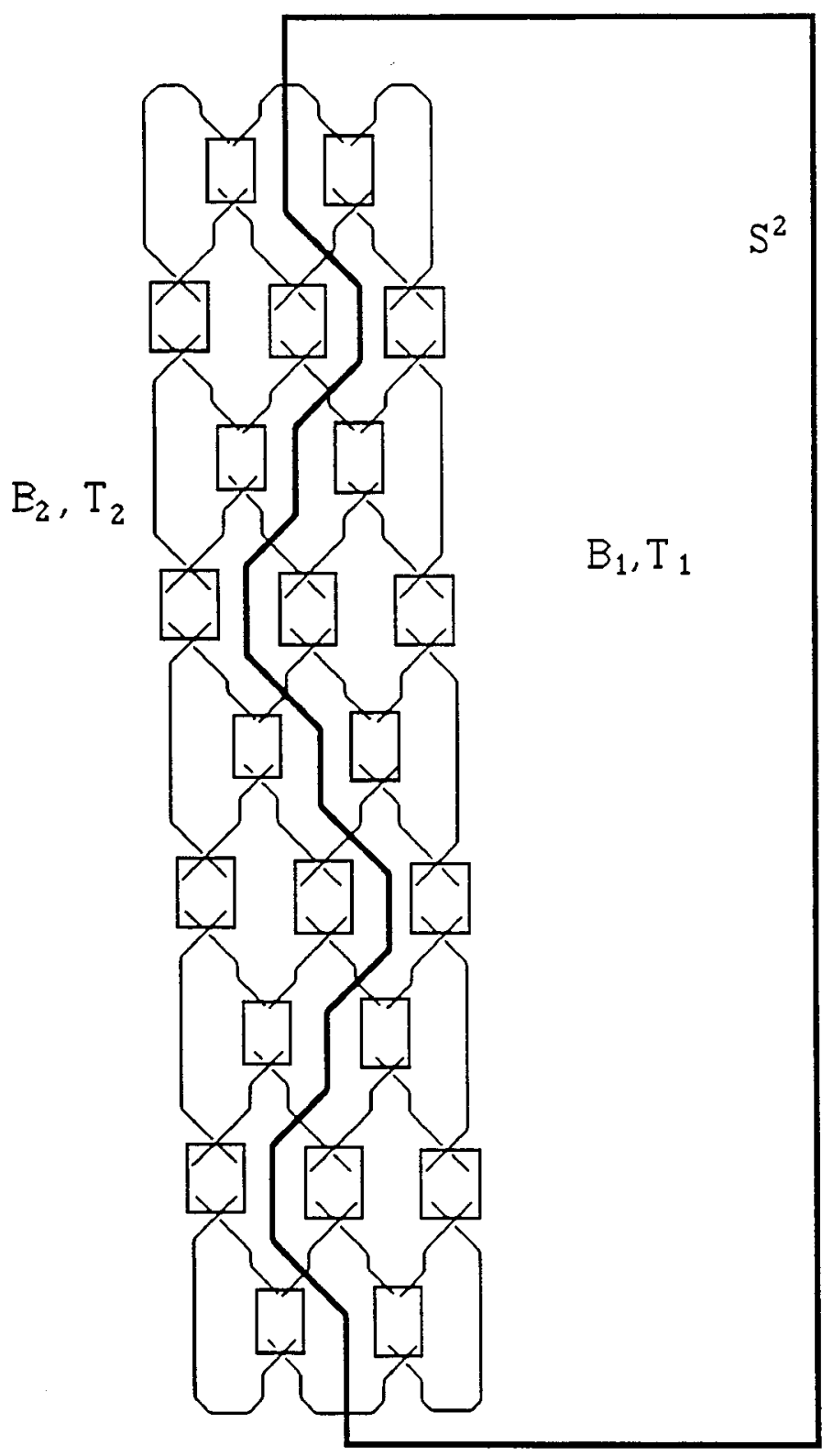

Figure 2

the top and bottom bridges and it separates an odd numbered row so that there is a box of crossings in each tangle and separates an even numbered row of three boxes of crossings into two boxes in one tangle and one box in the other alternately. The intersection of the 2-sphere with the projection plane is indicated by $S^{2}$ in Figure 2, for $m=9, n=3$.

Remark 2.3. Note that the definition of a vertical 2-sphere gives two such spheres al when $n=3$, since the vertical sphere is determined by how it divides the first 
row of three boxes into a set of two boxes and a single box and there are two such choices. In general we can define $2 n-4$ vertical 2 -spheres in a $2 n$-plat.

Lemma 2.4. Let $K$ be a knot or link in a 6-plat projection with $m \geq 5$. Then for each tangle $\left(B_{k}, T_{k}\right), k=1,2$, determined by a vertical 2-sphere, there is a collection of properly embedded disks $d_{1}, \ldots, d_{r}$, each of which intersects $T_{k}$ in exactly two points. Furthermore, the disks $d_{1}, \ldots, d_{r}$ cut $\left(B_{k}, T_{k}\right), k=1,2$, into sub-tangles, each of which is homeomorphic, fixing the disks, to one of the seven sub-tangles as shown in Figure 3, up to rotation and reflection.

Remark 2.5. Note that the tangles of type $A^{*}, B^{*}, C^{*}, C^{* *}$ are isotopic to the tangles $A, B, C$, respectively, by an isotopy which fixes the disk $d_{i}$ (or disks in the case of tangles of type $C$ ).

Proof. Consider the vertical 2-sphere $S$ cutting the knot or link in the 6-plat configuration into the two tangles. It is clear that each time $S$ separates a row of three boxes of crossings into two boxes in one tangle and one box in the other, we can properly embed a disk $d_{i}$ in the tangle containing the single box so that $T$ will intersect $d_{i}$ in exactly two points $p_{1}^{i}, p_{2}^{i}$ and $\partial d_{i} \subset \partial B_{k}$. Now consider the tangle $\left(B_{1}, T_{1}\right)$. We can insert the disk $d_{1}$ below the second row of boxes and then insert a disk every four rows. Since the number of rows $m$ is always odd, the number of disks is $\left[\frac{m-2}{4}\right]+1$, where $[a]$ denotes the integer value of $a$. If the residue of $m \bmod 4$ is 3 , we will have a type $A$ sub-tangle at the top and at the bottom. If $m \bmod 4$ is 1 , we will have a type $A$ sub-tangle at the top and a type $B$ at the bottom. If the number of rows is equal to or greater than 7 the sub-tangles in the middle will be of type $C$. Note that sub-tangles of type $A$ and $B$ appear only at the top and bottom of $\left(B_{k}, T_{k}\right)$. In the case of the tangle $\left(B_{2}, T_{2}\right)$ we will have a sub-tangle of type $B$ at the top and if $m \bmod 4=1$, we will have a type $A$

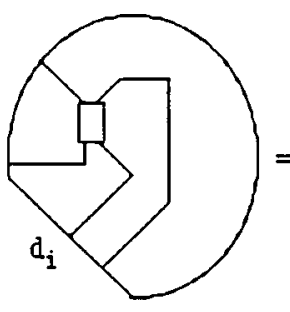

type A

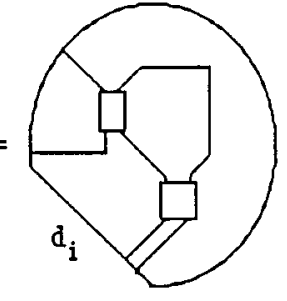

type $A^{*}$

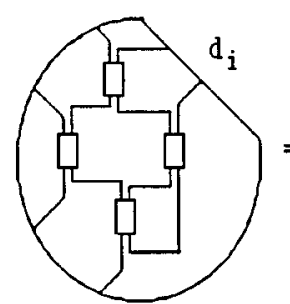

type B

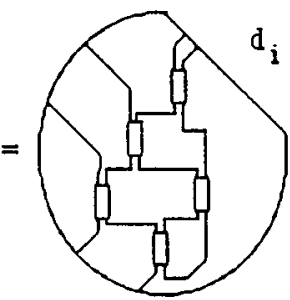

type $B^{*}$
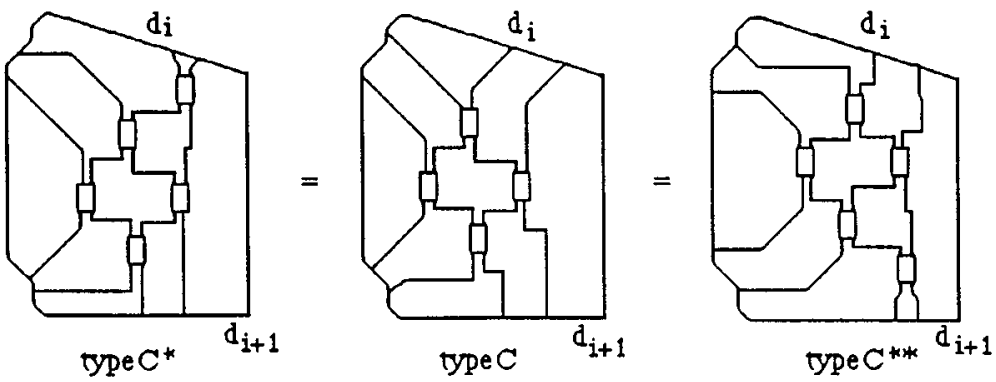

Figure 3 
sub-tangle at the bottom. If $m \bmod 4=3$, we will have a sub-tangle of type $B$ at the bottom. The rest of the sub-tangles are of type $C$. Altogether we have $\left[\frac{m}{4}\right]$ disks in this case. Now the claim follows by an easy induction argument on $m$.

By the "configuration of the strings" we mean the linking pattern of the various strings, i.e., which strings link and in what order. The configuration of the strings in the sub-tangles of type $A, B$ and $C$ is determined by the parity of the crossing numbers $a_{i, j}$ that they contain (see Lemma 2.4 and Figure 3). Each sub-tangle of type $B$ or $C$ contains four such crossing numbers and is thus determined by four integers $a_{1}, a_{2}, a_{3}, a_{4}$. There are sixteen sub-tangles of type $B,(C)$ denoted by $B(1)-B(16),(C(1)-C(16))$.

Lemma 2.6. Let $\left(B, t_{1}, t_{2}\right)=\left(D^{2}, p_{1}, p_{2}\right) \times I$ be the trivial 2 -tangle. Then there is a unique disk separating the two strings up to an isotopy fixing a neighborhood of the strings.

Proof. Let $D_{1}$ be a disk in $\left(B, t_{1}, t_{2}\right)$ which is (diameter of $\left.D^{2}\right) \times I$. Let $D_{2}$ be a separating disk not isotopic to $D_{1}$ chosen so as to minimize the number of components of the intersection of all such disks with $D_{1}$. The intersection $D_{1} \cap D_{2}$ cannot contain simple closed curves since an innermost such curve on $D_{2}$ would bound a 3 -ball together with the disk on $D_{1}$. This 3-ball cannot contain the string since it does not intersect $\partial B$. By the irreducibility of $B^{3}$ we can isotope $D_{2}$ to reduce the number of components of $D_{1} \cap D_{2}$ contradicting the choice of $D_{2}$. Hence we can assume that $D_{1} \cap D_{2}$ is composed of arcs. Note that $D_{1}$ cuts $\left(B, t_{1}, t_{2}\right)$ into two 3-balls $B_{1}, B_{2}$ each of which contains only one string. Let $D^{\prime} \subset D_{2}$ be an outermost disk in $D_{2}$ cut off by an arc of $D_{1} \cap D_{2}$. We may assume that $D^{\prime}$ is contained in, say, $B_{1}$. Now $D^{\prime}$ cuts $B_{1}$ into two 3 -balls, one of which does not contain the string $t_{1}$. We can now isotope $D_{2}$ off that 3 -ball to reduce the number of components of intersection of $D_{1} \cap D_{2}$ by an isotopy fixing a neighborhood of both strings. Again this is in contradiction to the choice of $D_{2}$ and proves the claim.

\section{Essential tangles}

In this section we prove a special case of Theorem 1.1 for knots or links admitting a 6-plat projection. Throughout this section we assume that all the conditions required in Theorem 1.1 are satisfied by all knots and links $K$.

Definition 3.1. An arc in one of the disks $d_{i}$ will be called essential if it cannot be isotoped into $\partial d_{i}$ by an isotopy fixing the points $p_{1}, p_{2}$. Otherwise, it will be called inessential.

Definition 3.2. An embedded disk $D \subset(B-T)$ will be called a compressing disk for the tangle $(B, T)$ if $\partial D \subset \partial B-N(T)$ represents a nontrivial element in $\pi_{1}(\partial B-N(T))$.

Proposition 3.3. Let $\mathcal{D}$ be a compressing disk for the tangle $\left(B_{k}, T_{k}\right), k=1,2$, chosen so that $\left|\mathcal{D} \cap\left\{\cup d_{i}\right\}\right|$, the number of components in the intersection $\mathcal{D} \cap\left\{\cup d_{i}\right\}$, is minimal. Then the set $\left\{\mathcal{D} \cap\left\{\cup d_{i}\right\}\right\}$ is not empty and contains no simple closed curves or inessential arcs.

In order to prove the proposition we need the following lemma:

Lemma 3.4. Every nontrivial simple closed curve $\sigma$ on the boundary of a subtangle $(B, T)$ of type $A, B$ or $C$ which misses the disks $d_{i}$ is nontrivial in the 
sub-tangle. In particular, the boundary curves $\partial d_{i}$, of the disks $d_{i}$, in the tangles $\left(B_{k}, T_{k}\right), k=1,2$, are essential curves in $B_{k}-T_{k}$.

Proof. Suppose, in contradiction, that some nontrivial curve $\sigma$ on the boundary of a sub-tangle $(B, T)$ which misses the disks $d_{i}$ bounds a disk $\Delta$ contained in the sub-tangle.

Case 1 . The disk $\Delta$ cannot be contained in a sub-tangle $(B, T)$ of type $A$ : If the number of crossings $a_{1}$ in $(B, T)$ is odd, the curve $\sigma$, being nontrivial, bounds a disk $B^{*}$ on $\partial B-d_{i}$ containing at least one endpoint of at least one of the strings. Since $a_{1}$ is odd, the disk $B^{*}$ cannot contain the other endpoint which is on $d_{i}$ and hence $\Delta$ separates this string. This is a contradiction as $\Delta \cap T=\varnothing$. If $a_{1}$ is even, then $\Delta$ must separate the sub-tangle into two 3 -balls each containing a string. Consider a small arc $\alpha$ on $d_{i}$ connecting the two endpoints of the string $t_{1}$ which has both endpoints on $d_{i}$. Since $\left|a_{1}\right|>1$, the simple closed curve $t_{1} \cup \alpha$ is homologous to $\left[a_{1} / 2\right] \neq 0 \in H_{1}\left(B-N\left(t_{2}\right)\right)$, where $t_{2}$ is the other string in $(B, T)$. Hence $\Delta$ cannot separate $t_{1}$ from $t_{2}$, a contradiction.

Case 2. The disk $\Delta$ cannot be contained in a sub-tangle $(B, T)$ of type $B$ : Consider the first five of sixteen sub-tangles of type $B$ denoted by $B(1)-B(5)$ as indicated in Figure 4, where "o" indicates odd and "e" indicates even crossing number $a_{i}$.

In the sub-tangles of type $B(1)-B(5)$ the two endpoints $p_{1}^{1}, p_{2}^{1}$ on the disk $d_{i}$ belong to the same string $t_{1}$. The disk $\Delta$ must therefore separate the string $t_{1}$ from the string $t_{2}$ or $t_{3}$ (or both). Since $\Delta \cap$ int $d_{i}=\varnothing$, we can connect the endpoints of $t_{1}$ by an arc $\alpha$ on $d_{i}$ pushed slightly into the interior of $B$. We denote these modified sub-tangles, which consist of a 3 -ball $B$ containing two properly embedded arcs and a simple closed curve $t_{1} \cup \alpha$, by $B^{\prime}(1)-B^{\prime}(5)$ respectively. Therefore, if a disk $\Delta$ exists in a sub-tangle of type $B(1)-B(5)$, it must also exist in the modified subtangle of type $B^{\prime}(1)-B^{\prime}(5)$.

The curve $\sigma=\partial \Delta$ bounds a disk $B^{*}$ on $\partial B-d_{i}$ and $B^{*}$ must contain some endpoint of a string that $\Delta$ separates from $t_{1}$ and hence it must contain both endpoints of that string. If the disk $B^{*}$ contains $\partial t_{2}\left(\partial t_{3}\right)$, we can connect the endpoints of the string $t_{2}\left(t_{3}\right)$ by an $\operatorname{arc} \beta(\gamma)$ in $B^{*}$.

If we can separate $t_{2}$ from $t_{1}$ by a disk $\Delta$, then we can also do this in the absence of $t_{3}$. So we can omit $t_{3}$ and after connecting the endpoints of $t_{1}$ and $t_{2}$ by arcs $\alpha$ and $\beta$, respectively, we obtain a 2-bridge link: $L(\alpha, \beta)=\left(t_{1} \cup \alpha\right) \cup\left(t_{2} \cup \beta\right)$. In cases $B^{\prime}(1), B^{\prime}(2)$ and $B^{\prime}(5)$ the link $L(\alpha, \beta)$ is determined by the rational number $\alpha / \beta=\left[a_{1},-a_{3}, a_{4}\right]$, where $\left[a_{1},-a_{3}, a_{4}\right]$ is the continued fraction expansion. The separating disk $\Delta$ will be a half of a splitting sphere for the links. Since $\left|a_{i}\right|>1$,

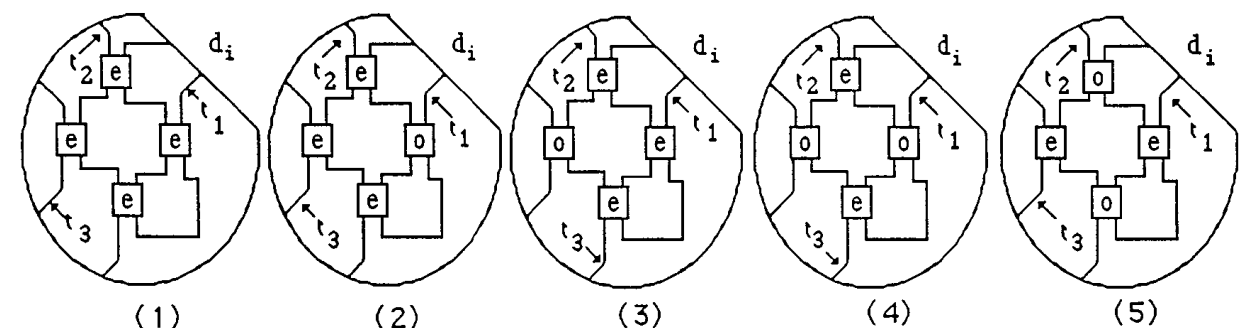

Figure 4 


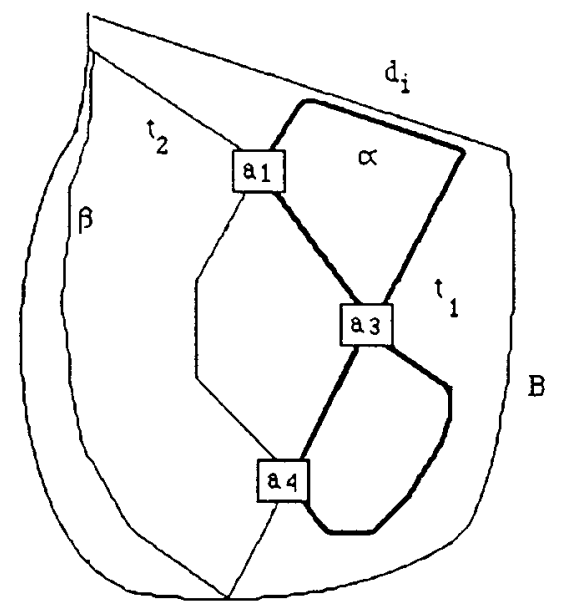

Figure 5

$i=1,3,4$, the link $L(\alpha, \beta)$ is not a trivial link and by definition of a 2 -bridge link it is not a split link. In cases $B^{\prime}(3)$ and $B^{\prime}(4)$ we obtain a torus link $L\left(2, a_{1}\right)$ which is also non-split since $\left|a_{i}\right|>1$. Hence we cannot have a disk $\Delta$ separating $t_{2}$ from $t_{1}$ (see, for example, Figure 5 for case $B^{\prime}(1)$ ).

Suppose now that we can separate $t_{3}$ from $t_{1}$ by a disk $\Delta$, then we can do this in the absence of the string $t_{2}$. As before, in cases $B(3), B(4)$ and $B(5)$ we can omit the string $t_{2}$ and connect the endpoints of $t_{1}$ and $t_{3}$ by arcs $\alpha$ and $\gamma$, respectively, to obtain torus links $L=\left(t_{1} \cup \alpha\right) \cup\left(t_{3} \cup \gamma\right)$ of the form $L\left(2, a_{4}\right), L\left(2, a_{4}\right)$ and $L\left(2, a_{2}\right)$ respectively. These links are non-split since $\left|a_{i}\right|>1$, so we cannot have a disk $\Delta$ separating $t_{3}$ from $t_{1}$ in these cases.

We still need to show that in cases $B(1)$ and $B(2)$ we cannot separate the string $t_{3}$ from the strings $t_{1} \cup t_{2}$ by a disk $\Delta$. The argument must be different since in the absence of $t_{2}$ we can separate $t_{3}$ from $t_{1}$ by a disk $\Delta$.

If there is a disk $\Delta$ in one of $B^{\prime}(1), B^{\prime}(2)$ which splits off the unknotted arc $t_{3}$, then it also cuts $B^{\prime}(i), i=1,2$, into two 3 -balls. One is a 3 -ball $B^{\#}$ with an unknotted arc $t_{3}$ and the other $B^{\# \#}$ is a 3 -ball containing the arc $t_{2}$ and the simple closed curve $t_{1} \cup \alpha$. Hence there is an arc $\gamma$ on the boundary of the $B^{\#}$ in the complement of the gluing disk $\Delta$ so that $\gamma \cup t_{3}$ bounds a disk in $B^{\#}$. This disk survives the gluing of $B^{\#}$ to $B^{\# \#}$ so the curve $\gamma \cup t_{3} \subset \partial B-d_{i}$ bounds a disk in $B-N\left(t_{1} \cup \alpha \cup t_{2}\right)$. Hence $\left[\gamma \cup t_{3}\right]=1$ in $\pi_{1}\left(B-N\left(t_{1} \cup \alpha \cup t_{2}\right)\right)$.

The $\operatorname{arc} \gamma$ is a path on the meridional annulus $\left(\partial B-\partial t_{2}\right)$ with the two endpoints of $t_{3}$ removed. Hence we can omit one endpoint $p$ of $t_{2}$ and consider $\gamma$ as a path on the disk $\partial B-N(p)$ connecting the endpoints of $t_{3}$ in the complement of a third point $p$. Such a path, which determines an element in the 3-braid group, can wind alternately between the endpoints of $t_{3}$ and the remaining endpoint of $t_{2}$. Thus the contribution of the $\gamma$ part of the simple closed curve $\gamma \cup t_{3}$ to the word $\left[\gamma \cup t_{3}\right]$ is $v^{m}$, where $v$ is the generator of $\pi_{1}\left(B-N\left(t_{1} \cup \alpha \cup t_{2}\right)\right)$ corresponding to the foot of the string $t_{2}$ and $m \in \mathbb{Z}$, as indicated in Figure 6 .

The $t_{3}$ part of the simple closed curve $\gamma \cup t_{3}$ contributes to $\left[\gamma \cup t_{3}\right]$ an element in $\pi\left(B-N\left(t_{1} \cup \alpha \cup t_{2}\right)\right)$ which depends on the linking of $t_{3}$ and $t_{1} \cup \alpha \cup t_{2}$. In both $B^{\prime}(1)$ and $B^{\prime}(2) a_{4}$ is even and hence $1=\left[\gamma \cup t_{3}\right]=z^{k_{2}} v^{m}$, where $z=(u v)^{-k_{4}} v(u v)^{+k_{4}}$ 


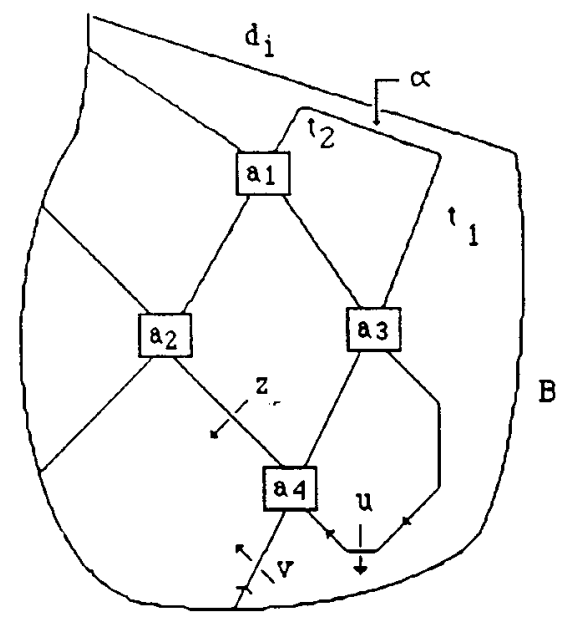

Figure 6

in case $B^{\prime}(1)$, or $z=\left(u^{-1} v\right)^{-k_{4}} v\left(u^{-1} v\right)^{+k_{4}}$ in case $B^{\prime}(2)$. If the word $z^{k_{2}} v^{m}$ is a relator in a group of a 2-bridge link, which abelianizes to $\mathbb{Z} \oplus \mathbb{Z}$, we must have $m=-k_{2}$.

Note that in each of $B^{\prime}(1), B^{\prime}(2)$ the fundamental group $\pi_{1}\left(B-N\left(t_{1} \cup \alpha \cup t_{2}\right)\right)$ is the group of a 2 -bridge link $L(\alpha, \beta), \alpha / \beta=\left[a_{1},-a_{3}, a_{4}\right]$. Since $\left|a_{i}\right|>1$, these links are never of the form $L(\alpha, 1)$ i.e., they are not torus links. Hence their complements $S^{3}-N(L(\alpha, \beta))=B-N\left(t_{1} \cup \alpha \cup t_{2}\right)$ are hyperbolic.

Any 2-bridge knot or link group $G$ has a presentation, with generators as indicated in Figure 6, as follows (see [BZ], p. 208):

$$
\left.\left.G=\langle u, v| u^{-1} L^{-1} v L\right]\right\rangle \text { where } L=u^{\varepsilon_{1}} v^{\varepsilon_{2}} \cdots u^{\varepsilon_{\alpha-2}} v^{\varepsilon_{\alpha-1}}
$$

when $\alpha=1 \bmod 2$ i.e., $L(\alpha, \beta)$ is a knot and

$$
\left.\left.G=\langle u, v| v^{-1} L^{-1} v L\right]\right\rangle \text { where } L=u^{\varepsilon_{1}} v^{\varepsilon_{2}} \cdots v^{\varepsilon_{\alpha-2}} u^{\varepsilon_{\alpha-1}}
$$

when $\alpha=0 \bmod 2$ i.e., $L(\alpha, \beta)$ is a link, where $\varepsilon_{i}=(-1)^{[i \beta / \alpha]}$ and $[a]$ is the integral part of $a$. Now set $a_{i}=2 k_{i}$ if $a_{i}$ is even and if $a_{i}$ is odd set $a_{i}=2 k_{i} \pm 1$, depending on whether $a_{i}$ is positive or negative.

We need the following lemma:

Lemma 3.5. The word $z^{k_{2}} v^{-k_{2}}$ is not a relation in $\pi_{1}\left(S^{3}-N(L(\alpha, \beta))\right)$.

Proof. Assume first that $\left|k_{2}\right|>1$. In this case the subgroup $\langle v, z\rangle$ generated by $v$ and $z$ in $G=\pi_{1}\left(S^{3}-N(L(\alpha, \beta))\right)$ is non-cyclic. If it was cyclic there would be an element $w \in G$ so that $v=w^{r}$ and $z=w^{s}$. This would contradict the fact that the images of both $v$ and $z$ in the abelianization of $G$ are generators of a $\mathbb{Z}$ factor, unless $r=s$. If $r=s$, then $v=z$ which contradicts our assumption $\left|k_{2}\right|>1$. Hence the group $\langle v, z\rangle$ has a nontrivial center and by Lemma VI.1.5 of [JS] the manifold $M=B-N\left(t_{1} \cup \alpha \cup t_{2}\right)$ must contain a nontrivial Seifert characteristic variety. This is a contradiction since $M$ is a hyperbolic manifold. (See also Theorem VI.4.1 of $[\mathrm{Sh}]$.

If $\left|k_{2}\right|=1$, then $v=(u v)^{-k_{4}} v(u v)^{+k_{4}}$ or $v=\left(u^{-1} v\right)^{-k_{4}} v\left(u^{-1} v\right)^{+k_{4}}$. This is the relation of a $\left(2, k_{4}\right)$-torus link group $H$. Since $\{u, v\}$ is a generating set for the 
group $G$, it follows that $G$ is a quotient of $H$. Recall that $H=\left\langle x, y \mid x^{2}=y^{k_{4}}\right\rangle$. Since $G$ is hyperbolic, the centralizer of $H$ must be mapped to $\{e\} \in G$. It follows that $G$ contains a quotient of $\left\langle x, y \mid x^{2}=y^{k_{4}}\right\rangle /$ Center $=\mathbb{Z}_{2} * \mathbb{Z}_{k_{4}}$ and all elements of finite order must be in the kernel since $G$ does not contain such elements. This would imply that the images of both $x$ and $y$ are in the kernel. However, the images of $x$ and $y$ generate the nontrivial group $G$. This is a contradiction and we cannot have a relation $z=v$ in $G$.

Hence we cannot have a disk $\Delta$ separating $t_{3}$ from $t_{1}$ in sub-tangles of type $B^{\prime}(1)$ and $B^{\prime}(2)$. Consequently, we cannot have a disk $\Delta$ in sub-tangles of type $B(1)-B(5)$.

Consider now the remaining eleven sub-tangles of type $B$ denoted by $B(6)-B(16)$ as indicated in Figures 10 and 11 and where $B(16)$ denotes the tangle where all $a_{i}$, $i=1, \ldots, 4$, are odd. Note that in all the tangles $B(6)-B(16)$ the strings $t_{1}$ and $t_{2}$ have endpoints on the disk $d_{i}$ so as before, since the disk $\Delta$ if it exists misses the disk $d_{i}$, we can connect the endpoints of $t_{1}$ and $t_{2}$ by an $\operatorname{arc} \alpha$ to obtain one string $t=t_{1} \cup \alpha \cup t_{2}$. We denote the new 2-tangles by $B^{\prime}(6)-B^{\prime}(16)$ and the existence of a disk $\Delta$ in $B(6)-B(16)$ implies the existence of $\Delta$ in $B^{\prime}(6)-B^{\prime}(16)$.

We can cut each of the sub-tangles $B^{\prime}(6)-B^{\prime}(16)$ by a properly embedded disk $\Delta_{1}$ into two 2-tangles $B_{1}$ and $B_{2}$ separating the $a_{2}$ twists from the rest of the tangle. We can then further cut the 2-tangle $B_{2}$ by a properly embedded disk $\Delta_{2}$ into two 2-tangles $B_{2}^{1}$ and $B_{2}^{2}$ separating the $a_{1}$ twists from the $a_{3}$ and $a_{4}$ twists as indicated in Figure 7. The tangles $B_{1}$ and $B_{2}^{1}$ are $a_{2}$ and $a_{1}$ twist tangles, respectively, in the terminology of [Wu 2] and the tangle $B_{2}^{2}$ is a rational tangle. All of $B_{1}, B_{2}^{1}$ and $B_{2}^{2}$ are atoroidal, i.e., the complement of the strings does not contain essential tori, since the complements of the strings are handlebodies. By Lemma 3.2 of [Wu 2] the tangle $B_{2}$ is atoroidal and a second application of the lemma for $B_{2}$ and $B_{1}$ shows that each of $B^{\prime}(6)-B^{\prime}(16)$ is atoroidal. Since $\left|a_{i, j}\right|>1$, none of $B^{\prime}(6)-B^{\prime}(15)$ is a rational tangle since if we remove $t_{3}$ the arc $t$ is knotted (i.e., there is no embedded disk $D$ so that $\partial D=t \cup \alpha$, where $\alpha$ is an $\operatorname{arc}$ on $\partial B$ ). It now follows from Lemma 3.1 of [Wu 2] that $\partial B-N\left(t \cup t_{3}\right)$ is incompressible. The tangle $B^{\prime}(16)$ does not contain a compressing disk by Lemma 3.3 of [Wu 2], since it is a sum of atoroidal tangles, neither of which is a 2-twist tangle (since in the case the $a_{i}$ 's are all odd). Therefore there is no compressing disk $\Delta$ in $B^{\prime}(6)-B^{\prime}(16)$ and hence there is none in $B(6)-B(16)$. This finishes the proof of Case 2.

Case 3 . The disk $\Delta$ cannot be contained in a sub-tangle $(B, T)$ of type $\mathrm{C}$ : In this case there are also sixteen different sub-tangles depending on the parity of the $a_{i}$ 's. Suppose that $(B, T)$ is of type $C(j)$. If $\sigma=\partial \Delta$ is a non-trivial simple closed curve in the annulus $\partial B-\left(\left(d_{i} \cup d_{i+1}\right)\right.$ less $(\cup$ endpoints of the strings $\left.)\right)$, then either it is parallel to the core of the annulus (if the strings are not fixed) or it bounds a disk on $\partial B$.

Suppose first that $\sigma$ is parallel to the core of the annulus. The curve $\sigma$ separates $\partial B$ into two disks $D_{i}, D_{i+1}$ containing the disks $d_{i}, d_{i+1}$ respectively. The disk $D_{i}\left(D_{i+1}\right)$ must contain all endpoints of the strings meeting the disk $d_{i}\left(d_{i+1}\right)$. Otherwise, the disk $\Delta$ will separate the endpoints of at least one of the strings, which clearly cannot happen. Thus $\sigma$ will remain nontrivial in $\partial B^{\prime}$-(strings), where $B^{\prime}$ is obtained from $B$ by connecting the endpoints of the strings on $d_{i+1}$ by a small arc and pushing this arc into the interior of $B$. We obtain a new tangle which is the same as $B(j)$ and the proof follows from that case. 


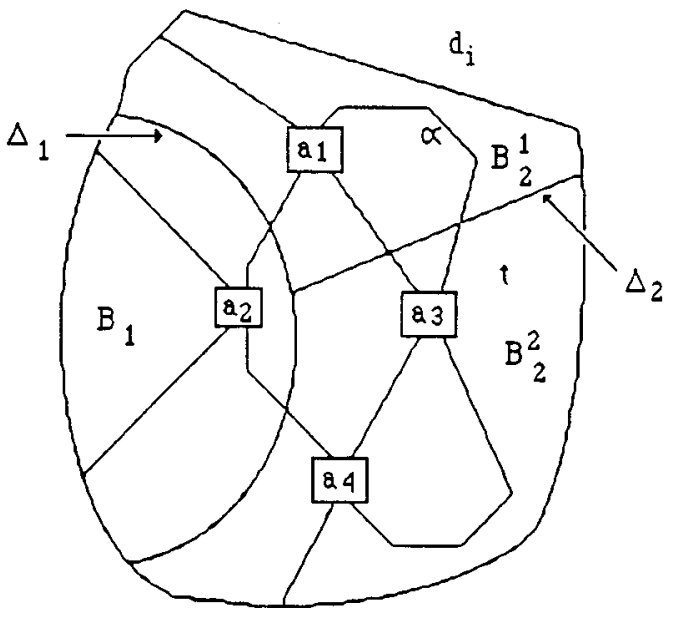

FIGURE 7

If $\sigma$ bounds a disk $B^{*}$ on the annulus, then we can connect the endpoints of the strings on the disk $d_{i}$ by an arc in $d_{i}$ and the curve $\sigma$ will continue to be nontrivial in the new tangle, since both $B^{*}$ and $\partial B-\left(B^{*} \cup d_{i}\right)$ contain endpoints of $T$. Again we obtain a new tangle which is the same as $B(j)$ and this completes the proof.

Proof of Proposition 3.3. If $\mathcal{D} \cap\left\{\cup d_{j}\right\}$ is empty, then $\mathcal{D}$ is contained on one of the sub-tangles of type $A, B$ or $C$. However, if this happens, then $\partial \mathcal{D}$ is a nontrivial loop on $\partial B$ which does not meet $d_{i}$ and bounds a disk $\Delta$ in the sub-tangle. By Lemma 3.4 this cannot happen. If $\mathcal{D} \cap\left\{\cup d_{j}\right\}$ contains simple closed curves, then it must contain a simple closed curve $\gamma$ bounding a sub-disk $d_{i}^{*} \subset d_{i}$, for some disk $d_{i}$. The disk $d_{i}^{*}$ must contain both intersection points of $T \cap d_{i}$ since if $d_{i}^{*} \cap T$ is empty, we can reduce the number of components in the intersection $\mathcal{D} \cap\left\{\cup d_{j}\right\}$ violating the choice of $\mathcal{D}$ and if $d_{i}^{*} \cap T$ contains only one point, we obtain a 2-sphere intersecting $K$ once. Hence $\gamma$ is isotopic to $\partial d_{i}$ and is compressible in contradiction to Lemma 3.4.

If any of the arcs of $\mathcal{D} \cap\left\{\cup d_{j}\right\}$ is inessential, we can replace $\mathcal{D}$ by a disk $\mathcal{D}^{*}$ with a smaller number of components in the intersection. This contradicts the choice of $\mathcal{D}$ and finishes the proof of Proposition 3.3.

Corollary 3.6. An outermost disk $E \subset \mathcal{D}$, cut by $\mathcal{D} \cap\left\{\cup d_{j}\right\}$, must intersect $\left\{\cup d_{j}\right\}$ in a single arc $\rho \subset d_{i}$, for some $i \in\{1, \ldots, r\}$. The arc $\rho$ must separate the two points of $T_{k} \cap d_{i}, k=1,2$, and hence $E$ separates the corresponding strings in the corresponding sub-tangle of type $A, B$ or $C$.

Proof. By Proposition 3.3 every intersection arc of $\mathcal{D} \cap\left\{\cup d_{j}\right\}$ must be an essential arc in the disk $d_{j}$ with the points $p_{1}^{j}, p_{2}^{j}$ removed and since $E \subset \mathcal{D}$ is outermost it must intersect some $d_{i}$ in a single arc $\rho$.

Theorem 3.7. Let $K$ be a knot in a 6-plat projection. If $m \geq 5$ and $\left|a_{i, j}\right|>1$, for each $i$ and $j$, then the tangles $\left(B_{1}, T_{1}\right)$ and $\left(B_{2}, T_{2}\right)$ are essential.

Proof. Assume, in contradiction, that there is some compressing disk $\mathcal{D}$ for $\left(B_{k}, T_{k}\right)$, $k=1,2$. We can choose a disk $\mathcal{D}$ so as to minimize the number of components 


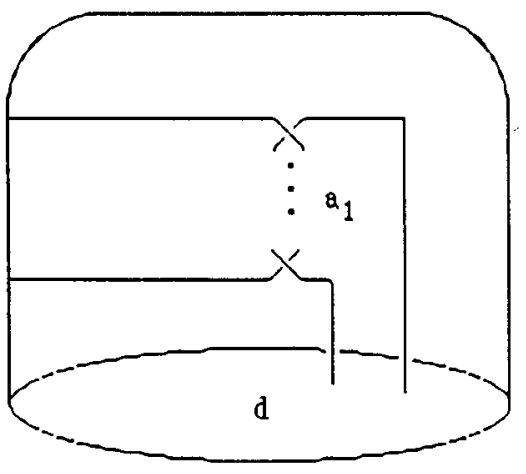

(a)

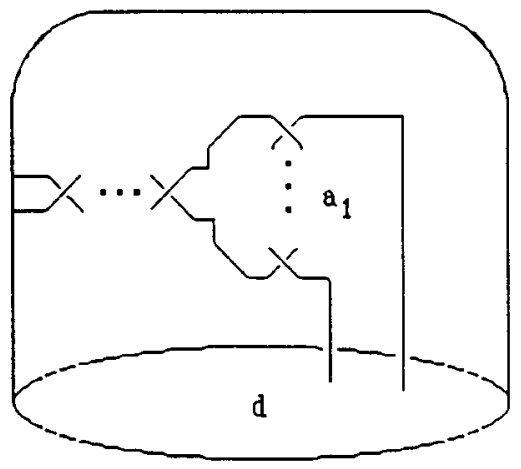

(b)

FiguRE 8

in the intersection of $\mathcal{D} \cap\left\{\cup d_{j}\right\}$. We may now assume, by Proposition 3.3, that $\mathcal{D} \cap\left\{\cup d_{j}\right\}$ contains no simple closed curves or inessential arcs. By Corollary 3.6 any outermost disk $E \subset \mathcal{D}$ must intersect a disk $d_{i}$ in a single arc $\rho$ separating the two points $p_{1}^{i}, p_{2}^{i}$ and is contained in a single sub-tangle.

It is sufficient to consider only sub-tangles of type $A$ or $B$. This is because the disk $E$ cannot intersect both the top disk $d_{i}$ and the bottom disk $d_{i+1}$ in a subtangle of type $C$. Hence if a disk $E$ like that exists in a sub-tangle of type $C$ it will also exist in the sub-tangle after we connect the points $p_{1}^{i}, p_{2}^{i}$, on the disk not met by $E$, by a small arc on the disk and then push it slightly into the interior of the 3 -ball. The resulting tangle, up to rotation, is a sub-tangle of type $B$.

Case 1. Assume that $E$ is contained in a sub-tangle of type $A$. The crossing number $a_{1}$ must be odd, since if $a_{1}$ is even, then $p_{1}^{1}, p_{2}^{1}$ belong to the same string and cannot be separated by $E$. Assume that $a_{1}$ is odd. The sub-tangle of type $A$ is isotopic to a tangle as in Figure 8(a) and after an isotopy (not fixing the strings) to a tangle as in Figure 8(b). The boundary of the disk $E$ is a union of two $\operatorname{arcs} \sigma \cup \rho$ where $\sigma$ is an arc on the twice punctured disk $\partial B-d$. If $\sigma$ is not an essential arc on $\partial B-d$, then we have a 2 -sphere intersecting the link $L$ once. Hence $\sigma$ is an arc separating the two endpoints of the strings on $\partial B-d$. By Lemma $2.6 \sigma$ is isotopic by an isotopy of $(B, T)$ twisting the endpoints of the strings in $\partial B-d$ about each other to the standard separating arc which we denote by $\sigma^{\prime}$. The isotopy above would induce an ambient isotopy of the disk $E$ to a new disk denoted by $\sigma^{\prime}$. After the isotopy the tangle looks as indicated in Figure 8(b). The curve $\partial E^{\prime}=\sigma^{\prime} \cup \rho$ separates the pairs of endpoints of the two strings. We can now connect the endpoints of each respective string by an arc to obtain a 2-bridge link as indicated by the thick lines in Figure 9. Hence the existence of the disk $E^{\prime}$ implies that this 2-bridge link is a split link. This is a contradiction and hence a disk $E$ does not exist.

Case 2. Assume that an outermost disk $E$ is contained in a sub-tangle of type $B$. We must consider all sixteen possible cases.

Sub-case (a). Consider the sub-tangles of type $B(1)-B(5)$ as in Figure 4 . In these sub-tangles the two endpoints $p_{1}, p_{2}$ on $d_{i}$ belong to the same string. Since the disk $E$ would have to separate them, such a disk cannot exist. 


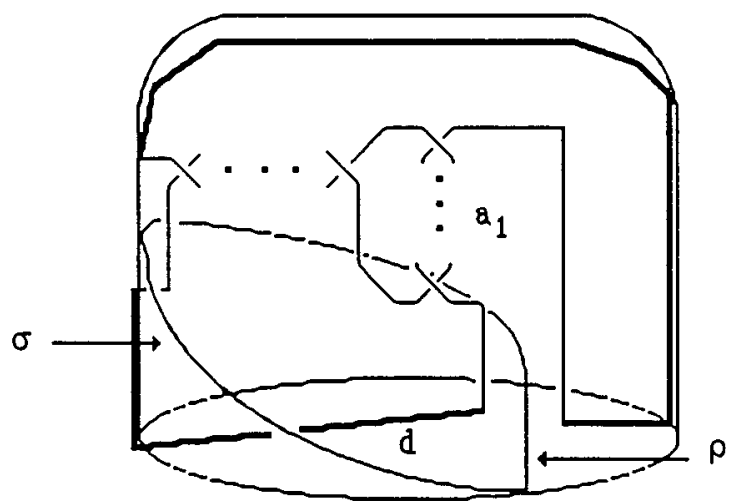

Figure 9

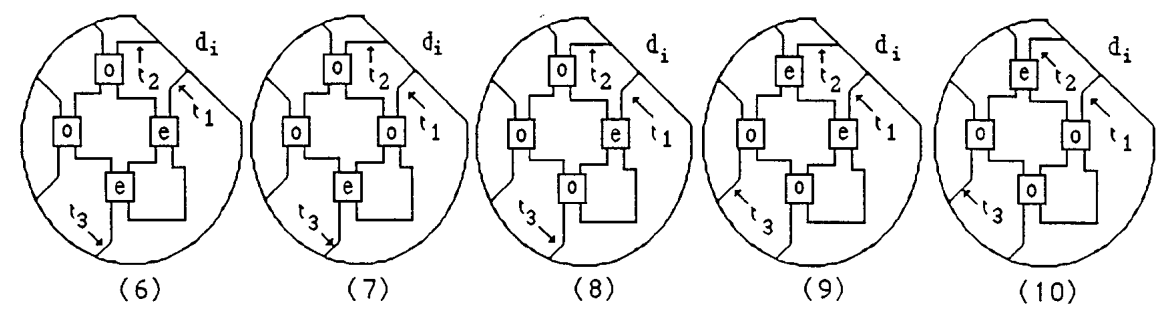

FiguRE 10

Sub-case (b). Consider the sub-tangles $B(6)-B(10)$ as in Figure 10.

The disk $E$ if it exists must also separate the strings $t_{1}$ and $t_{2}$ in the absence of $t_{3}$. So omit the string $t_{3}$ from all sub-tangles. Now, in sub-tangles (6) and (7) we can twist the interior of the string $t_{1}$, fixing the endpoints of the strings, to get rid of the $a_{3}$ crossings. In sub-tangles (9) and (10) we can twist about the disk $d_{i}$, fixing its boundary, to also get rid of the $a_{3}$ crossings. In all cases, including sub-tangle (8), we obtain a sub-tangle of type $A$. The existence of a disk $E$ in the sub-tangles of type (6)-(10) would imply the existence of such a disk in a sub-tangle of type $A$ which is a contradiction. Hence we cannot have such an outermost disk in these sub-tangles.

Sub-case (c). Consider the sub-tangles $B(11)-B(15)$ as in Figure 11. As in subcase (b) we can omit the $t_{3}$ string and the disk $E$, if it exists, must still separate the strings $t_{1}$ from $t_{2}$. By Lemma 3.8, which we prove below, the reduced word, represented by $\partial d_{i}$, in the free group $F(x, y)=\pi_{1}(H)=\pi_{1}\left(B-N\left(t_{1} \cup t_{2}\right)\right)$ has both generators occur at least twice if $\left|a_{i}\right|>1, i=1,3,4$. This implies that the intersection of the outermost disk $E$ and $d_{i}$ contains at least two arcs and rules out the existence of the disk $E$.

Lemma 3.8. Let $(B, T)$ be one of the sub-tangles of type $B(11)-B(15)$ with $\left|a_{i}\right|>$ $1, i=1,3,4$, in each case. Let $d(x, y)$ be a reduced word in the fundamental group $\pi_{1}\left(B-N\left(t_{1} \cup t_{2}\right)\right)=F(x, y)$ represented by the curve $\partial d_{i}$. Then $d(x, y)$ contains each of the generators $x$ and $y$, as indicated in Figure 12 below, at least twice.

Proof. Note that $d(x, y)$ is equal to $z^{-1} x$ as indicated in Figure 12. By checking all the different cases one sees that the word $z$ is a conjugate of the generator $y^{ \pm 1}$ by 


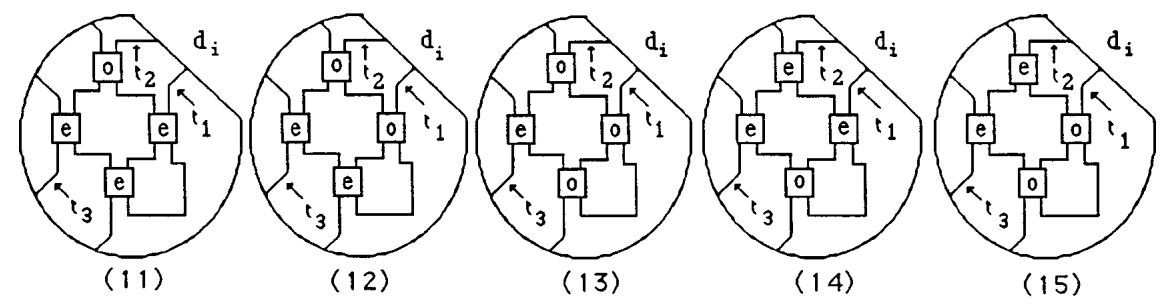

FIGURE 11

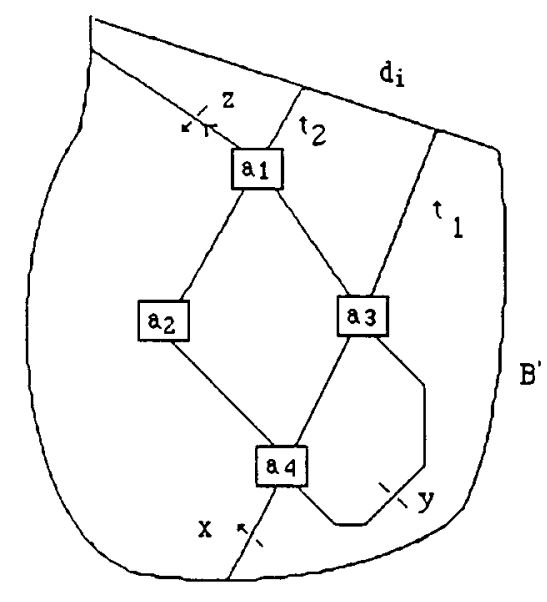

FIGURE 12

a word containing both generators $x$ and $y$. Hence the number of occurrences of $y$ in $z$ is greater than or equal to 3 unless $z=y$. If $\left|a_{i}\right|>1, i=1,3,4$, clearly that is not the case.

The number of occurrences of $x$ in $z$ must be even and thus greater than or equal to 2. If $x$ does not appear at the beginning or end of the word $z$, then the number of occurrences of $x$ in $z^{-1} x$ must be greater or equal to 3 . If $x$ does appear at the end of $z$ and $x z^{-1}$ has only one occurrence of $x$, then, for some $m \in \mathbb{Z}, z$ has the form $x^{-1} y^{m} y^{ \pm 1} y^{-m} x=x^{-1} y^{ \pm 1} x$. Again since $\left|a_{i}\right|>1, i=1,3,4$, it is clear that this is not the case.

Sub-case (d). Consider the sub-tangle of type $B(16)$. This case must be treated differently than the previous ones, since if we omit the $t_{3}$ string, we can find a disk $E$ with the required properties. We add a 1-tangle $\left(B, t^{\prime}\right)$ with a disk $d \subset \partial B$ to $B(16)$ along the disk $d_{i}$ in such a way so that the endpoints of the string $t^{\prime}$ get identified with the endpoints of the strings $t_{1}$ and $t_{2}$ on $d_{i}$. The 1 -tangle $\left(B, t^{\prime}\right)$ contains a disk $D$ intersecting $t^{\prime}$ in a single point and intersecting $d$ in a single arc (see Figure 13). After the gluing of the two tangles we obtain a 2-tangle which is isotopic to $B^{\prime}(16)$ as in the proof of Lemma 3.4

Assume now that the sub-tangle $B(16)$ contains an outermost disk $E \subset \mathcal{D}$ intersecting $d_{i}$ in a single arc. We can arrange the gluing map between $\left(B, t^{\prime}\right)$ and $B(16)$ so that the $\operatorname{arc} E \cap d_{i}$ is identified with the arc $D \cap d$. Hence the existence of a compressing disk $E$ in $B(16)$ implies the existence of an annulus $A=\left(D-N\left(t^{\prime}\right)\right) \cup E$ in 


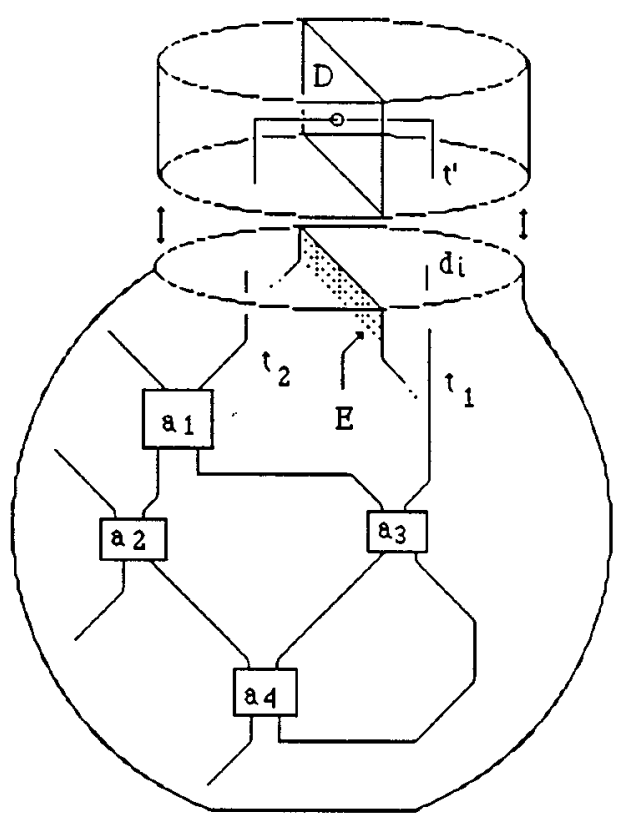

FiguRe 13

$B^{\prime}(16)$. Note that $A$ is incompressible, as one boundary component is a meridional curve, and not boundary parallel. Thus $A$ is an essential annulus.

Consider now a decomposition of $B^{\prime}(16)$ into sub-tangles $B_{1}$ and $B_{2}$ and then $B_{2}$ into $B_{2}^{1}$ and $B_{2}^{2}$ as in Figure 7 . Recall that the tangles $B_{1}$ and $B_{2}^{1}$ are $a_{2}$ and $a_{1}$ twist tangles, respectively, in the terminology of [Wu 2] and that the tangle $B_{2}^{2}$ is a rational angle. All the sub-tangles $B_{1}, B_{2}, B_{2}^{1}$ and $B_{2}^{2}$ are atoroidal as the complements of a regular neighborhood of the strings are handlebodies. By Lemma 3.4 of [Wu 2] one of the three following cases must hold, up to exchanging between $B_{1}$ and $B_{2}$ :

(i) The tangle $B_{1}$ is $\Delta$-annular.

(ii) The tangle $B_{1}$ is a $q$-twist tangle, with $q$ odd and the tangle $B_{2}$ is either a left torus tangle or a $p$-twist tangle with $|p| \geq 3$.

(iii) Both tangles $B_{i}$ are wrapping tangles with the unknotted string of $B_{1}$ glued to the unknotted string of $B_{2}$.

For the definitions of $\Delta$-annular, twist, torus and wrapping tangles see [Wu 2]. By Lemma 4.4 of [Wu 2] a nontrivial rational tangle is $\Delta$-annular if and only if it is a $2 n$-twist tangle with $|n| \geq 2$. A twist angle is, in particular, a rational tangle. Hence both $B_{1}$ and $B_{2}^{1}$ are not $\Delta$-annular since both $a_{2}$ and $a_{1}$ are odd. This rules out the first case (i) for $B_{1}$. Since no arc of $B_{2}$ has both endpoints on the gluing disk $\Delta_{1}$ between $B_{1}$ and $B_{2}$, the tangle $B_{2}$ is not a torus tangle. The tangle $B_{2}$ is clearly not a twist tangle, hence case (ii) is completely ruled out. Since both the strings in $B_{1}$ and $B_{2}$ are unknotted, neither $B_{1}$ and $B_{2}$ are wrapping tangles and hence case (iii) is completely ruled out. It remains to prove that $B_{2}$ is not $\Delta$-annular. 
If $B_{2}$ is $\Delta$-annular, then $B_{2}$ contains an essential annulus by Lemma 3.5 of [Wu 2]. We can now apply Lemma 3.4 of [Wu 2] to $B_{2}=B_{2}^{1}+B_{2}^{2}$. Neither of $B_{2}^{1}$ or $B_{2}^{2}$ is $\Delta$-annular since none of them is a $2 n$-twist tangle. This rules out case (i). Since $B_{2}^{1}$ and $B_{2}^{2}$ are not twist tangles and no one of them is a torus tangle, case (ii) is ruled out. As both arcs in $B_{2}^{1}$ and $B_{2}^{2}$ are unknotted, they are not wrapping tangles and case (iii) is ruled out as well. Therefore, the tangle $B^{\prime}(16)$ does not contain an essential annulus and hence we cannot have such a disk $E$ in a subtangle of type $B(16)$. This finishes the proof of the theorem.

Corollary 3.9. Let $K$ be a knot or a link in $S^{3}$ admitting a 6-plat projection, $m \geq 5$. If $\left|a_{i, j}\right|>1$, for each $i$ and $j$, then the two planar meridional surfaces $S-N(K)$ are essential in $S^{3}-N(K)$ and are not isotopic there.

Proof. By Theorem 3.7 each of the two vertical 2-spheres $S$ in the 6-plat knot $K$ cuts the pair $\left(S^{3}, K\right)$ into two essential sub-tangles $\left(B_{1}, T_{1}\right),\left(B_{2}, T_{2}\right)$ respectively. Hence $S^{3}-N(K)$ contains two meridonal essential planar surfaces $S-N(K)$. The two planar surfaces are not isotopic since the tangles they divide $\left(S^{3}, K\right)$ into are different.

Corollary 3.10. Let $K$ be a knot in $S^{3}$ admitting a 6-plat projection, $m \geq 5$. If $\left|a_{i, j}\right|>1$, for each $i$ and $j$, then $K$ is a nontrivial knot.

\section{General $2 n$-Plats}

In this section we will prove Theorem 1.1 for the general case. We consider knots or links $K \subset S^{3}$ in a $2 n$-plat projection where $n \geq 3$ and $m$, the number of rows of crossings as in Definition 1.1, is odd and greater than or equal to 5 . Let $S$ be a vertical 2-sphere (see Definition 2.2) which intersects the knot $K$ on the $n-1$ (counted from the left) top and bottom bridges and then separates periodically one box of crossings in the first three rows and two boxes of crossings in the following row (counted from the right). See Figure 14.

The 2 -sphere separates $\left(S^{3}, K\right)$ into two tangles $\left(B_{1}, T_{1}\right),\left(B_{2}, T_{2}\right)$ and we denote by $\left(B_{1}, T_{1}\right)$ the tangle containing the $n$th bridge. Note that if $n>3$, then $\left(B_{1}, T_{1}\right)$ is an essential tangle by Theorem 3.7 and if $n=3$, then so is $\left(B_{2}, T_{2}\right)$. Therefore, the goal of this section is to prove that with appropriate conditions the tangle $\left(B_{2}, T_{2}\right)$ is essential when $n>3$. Since the number of strings in the tangle $\left(B_{2}, T_{2}\right)$ is always greater than or equal to two, it is sufficient to prove that $\partial B_{2}$ is incompressible in the complement of $T_{2}$ (i.e., we do not have to worry about boundary compressibility).

Let $n$ be greater than or equal to 4 . Then tangle $\left(B_{2}, T_{2}\right)$ can be cut by a disk $D$ into two sub-tangles $\left(B_{2}^{1}, T_{2}^{1}\right)$ and $\left(B_{2}^{2}, T_{2}^{2}\right)$ defined as follows:

The boundary of $D$ is on $S$ and the disk $D$ cuts through the $(n-2)$ nd top and bottom bridges (counted from the left). In each row of boxes of crossings it cuts through the row one box further to the left than $S$ as indicated in Figure 15 (a) for $m=9$ and $n=5$. The tangle $\left(B_{2}, T_{2}\right)$ is the union of the tangles $\left(B_{2}^{1}, T_{2}^{1}\right)$ and $\left(B_{2}^{2}, T_{2}^{2}\right)$ along the disk $D$. Denote the "small" sub-tangle i.e., the one containing only one box of crossings from each row by $\left(B_{2}^{1}, T_{2}^{1}\right)$ (see Figure 15 (b)).

Note that there are $m-1$ disks $d_{1}, \ldots, d_{m-1}(m \geq 5$ being the number of rows of boxes of crossings) properly embedded in $\left(B_{2}^{1}, T_{2}^{1}\right)$. Each disk $d_{i}$ cuts the strings 


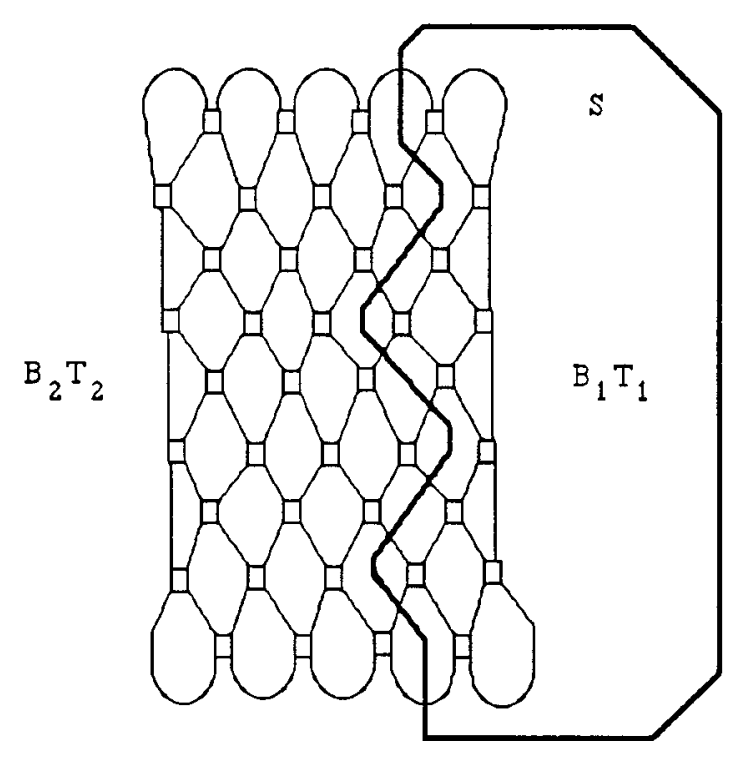

Figure 14

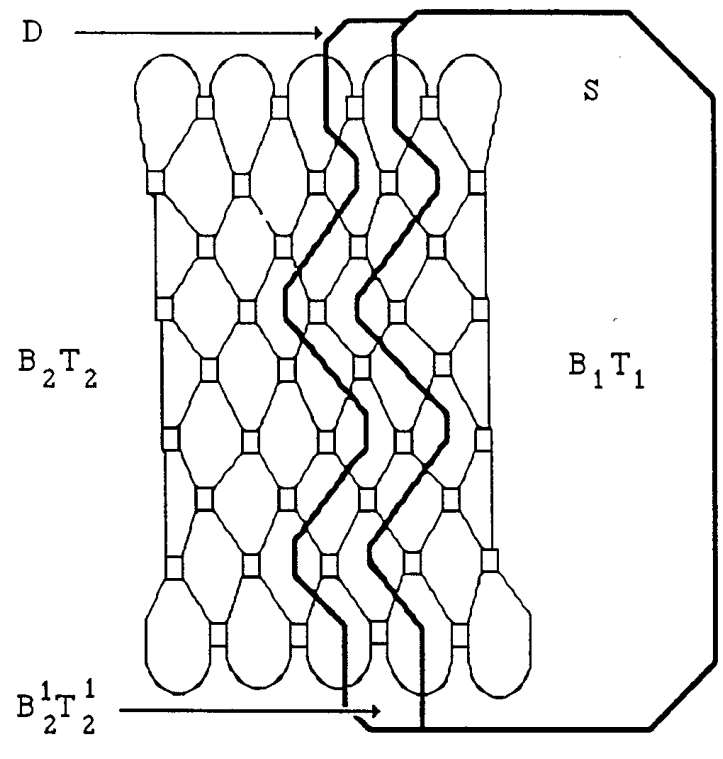

(3)

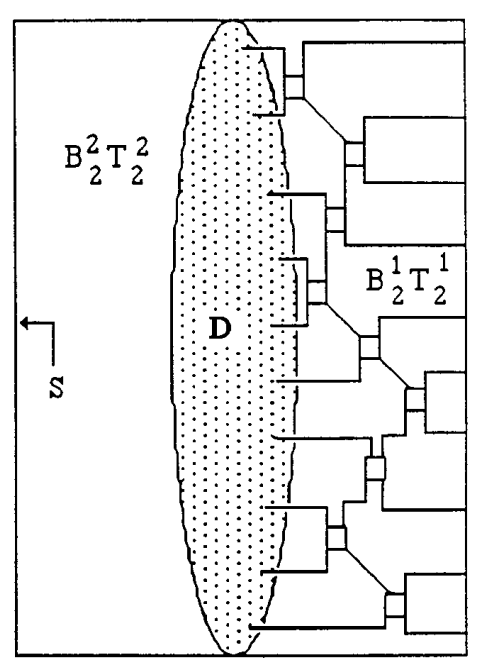

(b)

FiguRe 15

$T_{2}^{1}$ in exactly one point $p_{i}$. The disks $d_{1}, \ldots, d_{m-1}$ separate the tangle $\left(B_{2}^{1}, T_{2}^{1}\right)$ into $m$ sub-tangles $\left(B^{1}, T^{1}\right), \ldots,\left(B^{m}, T^{m}\right)$ (indicated in Figure 16).

We have the following lemma: 


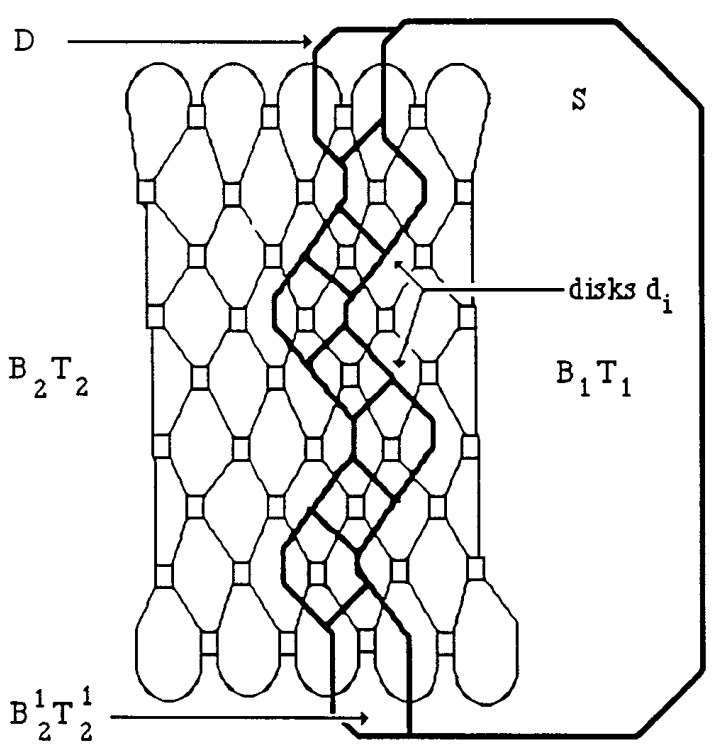

Figure 16

Lemma 4.1. Let $K$ be a knot or link in $S^{3}$ in a $2 n$-plat projection. Let $S, D$ and $d_{1}, \ldots, d_{m-1}$ be as above. Then each of the tangles $\left(B^{j}, T^{j}\right), j=1, \ldots, m$, is one of the following six types up to reflections:

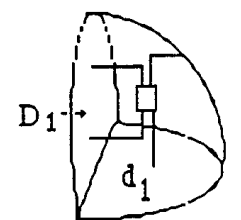

(1)

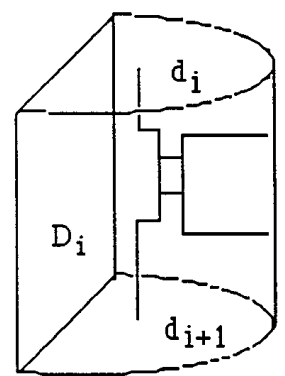

(3)

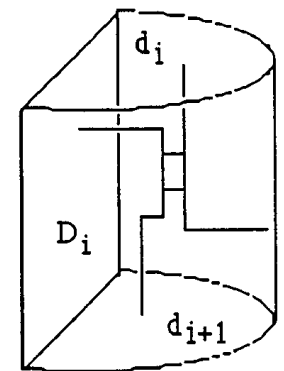

(4)

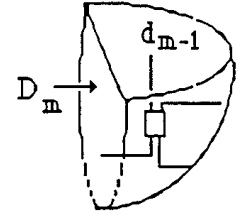

(2)

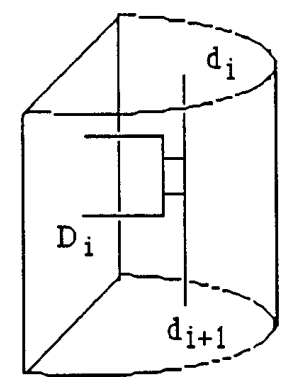

(5)

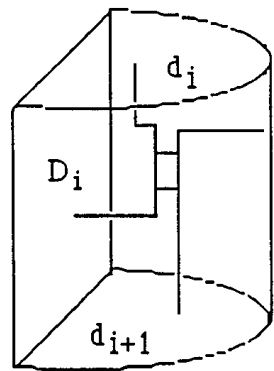

(6)

FiguRe 17

Proof. Consider the sub-tangles appearing at the "top" and "bottom" of $\left(B_{2}^{1}, T_{2}^{1}\right)$, i.e., $\left(B^{1}, T^{1}\right)$ and $\left(B^{m}, T^{m}\right)$. From the definition of the vertical 2 -sphere $S$ and 
the disk $D$ it follows that $\partial\left(B^{1}, T^{1}\right)$ and $\partial\left(B^{m}, T^{m}\right)$ is the union of three disks, namely $d_{i}$, a sub-disk $D_{i}$ of $D$ and a sub-disk $S_{i}$ of the disk $S^{*}=\partial\left(B_{2}^{1}, T_{2}^{1}\right)-D=$ $S \cap\left(B_{2}^{1}, T_{2}^{1}\right), i \in\{1, m\}$. So the type of tangle is determined by the configurations of the endpoints of the strings. We must always have one string on $d_{i}, i \in\{1, m\}$, so if we have two endpoints on $D_{i}$ and one on $S_{i}$, we have a sub-tangle of type (1) and if we have one endpoint on $D_{i}$ and two on $S_{i}$, we have a sub-tangle of type (2). Note that it follows from the definitions that the sub-tangle $\left(B^{1}, T^{1}\right)$ is always of type (1) but $\left(B^{m}, T^{m}\right)$ can be either type (1) or type (2), depending on $m$.

Consider now the sub-tangles appearing in the "middle" of $\left(B_{2}^{1}, T_{2}^{1}\right)$, i.e., $\left(B^{i}, T^{i}\right)$, $i=2, \ldots, m-1$. From the definition of the vertical 2 -sphere $S$ and the disk $D$ it follows that $\partial\left(B^{i}, T^{i}\right)$ is the union of four disks, namely $d_{i}, d_{i+1}$ a sub-disk $D_{i}$ of $D$ and a sub-disk $S_{i}$ of the disk $S^{*}=\partial\left(B_{2}^{1}, T_{2}^{1}\right)-D=S \cap\left(B_{2}^{1}, T_{2}^{1}\right), i=2, \ldots, m-1$. As before, the type of tangle is determined by the configurations of the endpoints of the strings. We must always have the endpoints of two strings on $d_{i}$ and $d_{i+1}$, one emanating from the top of the box of crossings and the other from the bottom. So, if we have two endpoints on $S_{i}$ and one on $D_{i}$, we have a sub-tangle of type (3). If we have two endpoints on $D_{i}$ and none on $S_{i}$, we have a sub-tangle of type (5). If we have an endpoint of one of the top strings on $D_{i}$ and an endpoint of one of the bottom strings $S_{i}$, we have a sub-tangle of type (4). Finally, if we have an endpoint of one of the bottom strings on $D_{i}$ and an endpoint of one of the top strings on $S_{i}$, we have a sub-tangle of type (6).

Lemma 4.2. Let $K$ be a knot or link in $S^{3}$ admitting a $2 n$-plat projection with $m \geq 5$ and $\left|a_{i, j}\right|>1$. Then there is no compressing disk $\Delta$ for the tangle $\left(B_{2}^{1}, T_{2}^{1}\right)$ such that $\partial \Delta$ is contained in $S^{*}=\partial\left(B_{2}^{1}, T_{2}^{1}\right)-D$ or in $D$.

Proof. Let $\Delta$ be a compressing disk for the tangle $\left(B_{2}^{1}, T_{2}^{1}\right)$ so that $\Delta \cap D=\varnothing$. Choose such a disk which minimizes the number of components of intersection of $\Delta \cap\left\{\cup d_{i}\right\}, i=1, \ldots, m-1$. Assume $\Delta \cap\left\{\cup d_{i}\right\}$ is empty. Note that $\partial \Delta$ is contained in $\partial\left(B_{2}^{1}, T_{2}^{1}\right)-D$ and bounds a disk $B^{*}$ there. Cases (1), (4), (5) and (6) of Lemma 4.1 cannot occur since then $B^{*}$ can contain only one or less endpoints of $T$ and this implies that either $B^{*} \cup \Delta$ is a 2 -sphere intersecting $K$ in one point or $\partial \Delta$ is trivial. Cases (2) and (3) cannot occur since both cases are trivial 2-tangles and in such 2-tangles a compressing disk is unique and does not miss $\partial\left(B_{2}^{1}, T_{2}^{1}\right)-D$. We may conclude that for each $i, \Delta \cap d_{i}$ must be a collection of arcs and simple closed curves. If $\Delta \cap d_{i}$ contains any simple closed curve $\gamma$, then an innermost $\gamma$ bounds a sub-disk $d_{i}^{*}$ of $d_{i}$. The disk $d_{i}^{*}$ cannot contain the point $p_{i}$ on $d_{i}$ since then we would have a 2 -sphere $d_{i}^{*} \cap$ (disk bounded by $\gamma$ on $\Delta$ ) intersecting the knot or link $K$ in a single point. However, if $d_{i}^{*}$ does not contain the point $p_{i}$ on $d_{i}$, then we can find a disk with a smaller number of components of intersection contrary to the choice of $\Delta$. We conclude that $\Delta \cap\left\{\cup d_{i}\right\}$ is a collection of arcs cutting $\Delta$ into sub-disks.

Let $E$ be an outermost sub-disk of $\Delta$. The disk $E$ is contained in a unique sub-tangle $\left(B^{i}, T^{i}\right), i=1, \ldots, m$, which is of type (1)-(6), by Lemma 4.1. The boundary of $E$ is a simple closed curve which consists of two $\operatorname{arcs} \alpha \cup \delta$ where $\alpha$ is an $\operatorname{arc}$ on $d_{i}$ and $\delta$ is an $\operatorname{arc}$ on $S^{*}$. Recall that we are assuming that $\partial E \cap D=\varnothing$.

Case 1. Assume that the outermost disk $E$ is contained in a sub-tangle of type (1) or (2). The curve $\partial E$ must separate $\partial\left(B^{i}, T^{i}\right), i \in\{1, m\}$ into two disks each containing exactly two endpoints of the strings in $\left(B^{i}, T^{i}\right)$. Otherwise, either the 
intersection of $\Delta$ with $\cup d_{i}$ is not minimal or we get a 2-sphere intersecting the knot or link $K$ in a single point. Furthermore, each pair of endpoints separated by $E$ must, of course, belong to the same string. Now connect each pair of endpoints by an arc on their respective disks to obtain a two component link. This link is a $\left(2, a_{1, n-2}\right)$ or a $\left(2, a_{m, n-2}\right)$-torus link which is not split as $\left|a_{i, j}\right|>1$. Hence a disk $E$ cannot exist in sub-tangles of type (1) or (2).

Case 2. Assume that the outermost disk $E$ is contained in a sub-tangle $\left(B^{i}, T^{i}\right)$ of types (3)-(6). The curve $\partial E$ cannot intersect both the disk $d_{i}$ and $d_{i+1}$ in the sub-tangle $\left(B^{i}, T^{i}\right)$ and also must separate $\partial\left(B^{i}, T^{i}\right)$ into two disks each containing exactly two endpoints belonging to the same string, as in case (1). This rules out the possibility of $\left(B^{i}, T^{i}\right)$ being of type (5) since the fact that two endpoints are contained in $D$ requires that any such disk $E$ must intersect both $d_{i}$ and $d_{i+1}$. For the remaining cases we can connect each pair of endpoints by an arc on their respective disks to obtain a two component link as before. This link is a $\left(2, a_{i, n-1}\right),\left(2, a_{i, n-2}\right)$ or $\left(2, a_{i, n-3}\right)$-torus link depending on $i, i=2, \ldots, m-1$, which is not split as $\left|a_{i, j}\right|>1$. Hence a disk $E$ cannot exist in sub-tangles of type (3), (4) or (6).

If we assume that $\partial \Delta$ is contained in $D$, a close observation shows that the same configurations of endpoints occur in the sub-disks of $\partial\left(B^{i}, T^{i}\right)$ separated by $\partial E$, as in the previous case. Therefore, we obtain a contradiction as before.

4.3. Proof of Theorem 1.1. Consider the vertical 2-sphere $S$ separating $\left(S^{3}, K\right)$ into two tangles $\left(B_{1}, T_{1}\right)$ and $\left(B_{2}, T_{2}\right)$. By Theorem 3.7 the tangle $\left(B_{1}, T_{1}\right)$ is essential. So in order to finish the proof we need to show that the tangle $\left(B_{2}, T_{2}\right)$ is essential for $n \geq 3$.

The proof will be by induction on $n$. For $n=3$ the tangle $\left(B_{2}, T_{2}\right)$ is essential by Theorem 3.7. Let $n$ be greater than 3 and assume that the tangle $\left(B_{2}, T_{2}\right)$ is essential for all knots or links $K$ in a $2 k$-plat projection with $m \geq 5$ and $\left|a_{i, j}\right|>1$, for each $i$ and $j$ and all $k \leq n-1$. Now consider the tangle $\left(B_{2}, T_{2}\right)$ for a knot or link $K$ with a $2 n$-plat projection satisfying the other conditions above.

Insert the disk $D$ into the tangle $\left(B_{2}, T_{2}\right)$ separating it into two sub-tangles $\left(B_{2}^{1}, T_{2}^{1}\right)$ and $\left(B_{2}^{2}, T_{2}^{2}\right)$. Assume that $\Delta$ is a compressing disk for $\left(B_{2}, T_{2}\right)$. The curve $\partial \Delta$ is contained in $\partial B_{2}-N\left(T_{2}\right)$ and, in fact, we can assume, by irreducibility, that $\partial \Delta \subset S^{*}$. Consider now such a disk $\Delta$ which minimizes the number of components of intersection with $D$. The intersection $\Delta \cap D$ must consist of essential simple closed curves in $\partial B_{2}-N\left(T_{2}\right)$. Otherwise, we get a contradiction to the choice of $\Delta$ as intersection minimizing. Let $\gamma$ be an innermost such curve on $\Delta$ which bounds a sub-disk $\Delta^{*}$ of $\Delta$. The disk $\Delta^{*}$ cannot be contained in $\left(B_{2}^{2}, T_{2}^{2}\right)$ since this would contradict the induction assumption that the tangle $\left(B_{2}^{2}, T_{2}^{2}\right)$ is essential. On the other hand, it is not contained in $\left(B_{2}^{1}, T_{2}^{1}\right)$ as $\gamma \subset D$ and this would contradict Lemma 4.2. Hence we cannot have such a disk $\Delta$ and the tangle $\left(B_{2}, T_{2}\right)$ is essential. Note that the arguments work for all vertical 2 -spheres by symmetry. So, in particular, all vertical 2 -spheres are essential and there are $2 \cdot(n-2)$ of them: Two for each bridge of the projection, except the end bridges, depending on the two possible partitions of the second row of twists. These planar surfaces are nonisotopic since the tangles $\left(B_{1}, T_{1}\right),\left(B_{2}, T_{2}\right)$ are different for the two choices of 2 -spheres.

Remark 4.3. Theorem 6.1 of [GR] uses Propositions 2.2.1 and 2.3.1 of [CGLS] in order to conclude the existence of a closed incompressible surface. Proposition 2.2.1 deals with the case that the essential meridional surface $F$ in the manifold $M$ is 
nonplanar and Proposition 2.3.1 deals with the case where $F$ is planar in which case we can conclude that the closed surface is a torus. However, the assumption in both propositions on $F$ is that $F$ is the surface with the minimal number of boundary components realizing the given boundary slope (see [CGLS], p. 267). The planar essential meridional surface $\partial B_{1}-N\left(T_{1}\right)$, as proved in the proof of Theorem 1.1, is not necessarily minimal in that sense. Hence we cannot decide whether $F$ is planar or not and cannot conclude anything directly as to the genus of the closed incompressible surface.

\section{Consequences}

In this section we will show that the condition $\left|a_{i, j}\right|>1$ can be weakened. It is an interesting question, therefore, what are the exact conditions required for a knot or link complement to contain an essential meridional planar surface. We will also describe some consequences of Theorem 1.1 and its proof.

Lemma 5.1. Let $K$ be a knot or link in a $2 n$-plat projection with $n \geq 3$ and $m \geq 5$. Assume that the following conditions hold:

1. $\left|a_{i, j}\right|=1$ or 0, for $i=2 \bmod 4, j=2 n$, or

2. $\left|a_{i, j}\right|=1$ or 0 , for $i=0 \bmod 4, j=1$, and

3. all other $a_{i, j}$ satisfy $\left|a_{i, j}\right|>1$.

Then $S^{3}-N(K)$ contains $n-2$ planar nonisotopic essential meridional surfaces. When $j=1$ in (1) and $j=n$ in (2), the other $n-2$ planar meridional surfaces are essential.

Proof. We can cut $K$ by two vertical 2 -spheres, so that the rightmost and leftmost tangles are homeomorphic, respectively, to the tangles $\left(B_{1}, T_{1}\right)$ and $\left(B_{2}, T_{2}\right)$ we obtained in the 6-plat case. (In the 6-plat case one vertical 2-sphere will suffice.) By Lemma 2.4 and Remark 2.5 the tangles $\left(B_{1}, T_{1}\right)$ and $\left(B_{2}, T_{2}\right)$, each, can be cut further into sub-tangles of type $A^{*}, B^{*}, C^{*}$ and $C^{* *}$ which are isotopic to subtangles of type $A, B$ and $C$ respectively. This isotopy twists each of the sub-tangles fixing the disks $d_{i}$ and in the process unwinds exactly the crossings $a_{i, j}$ satisfying conditions (1) and (2). Hence the sub-tangles satisfy the conditions of Theorem 3.7 independently of the values of these $a_{i, j}$ 's. It follows that the tangle components of $K$ isotopic to $\left(B_{1}, T_{1}\right)$ and $\left(B_{2}, T_{2}\right)$ of the 6 -plat case are essential. As in the proof of Theorem 1.1 this implies that the $\left(B_{1}, T_{1}\right)$ and $\left(B_{2}, T_{2}\right)$ components of $K$ are essential. By considering all possible vertical 2 -spheres which are "parallel" to the first two we see that $S^{3}-N(K)$ contains $n-2$ planar nonisotopic essential meridional surfaces. If we consider the case $j=1$, in (1) and $j=n$, in (2), we obtain the other $n-2$ planar nonisotopic essential meridional surfaces by a similar argument.

The following definition appears in [GR]. A knot or link $L$ in $S^{3}$ is an $n$-string composite if the pair $\left(S^{3}, L\right)$ can be expressed as the union of two essential $n$-string tangles and a tangle composite if it is an $n$-string composite for some $n$. If $K$ is not an $n$-string composite, then it is $n$-string prime.

Corollary 5.2. Let $K$ be a knot or link in a $2 n$-plat, $n \geq 3$ projection. If $m \geq 5$, $\left|a_{i, j}\right|>1$, for each $i$ and $j$, then $K$ is an $(m+1) / 2$-string composite in at least $2 n-4$ different ways. 
Proof. In each such knot or link $K$ there are $2 n-4$ vertical 2 -spheres and by the proof of Theorem 1.1 each such 2 -sphere divides $\left(S^{3}, K\right)$ into two essential $(m+1) / 2$-tangles.

Corollary 5.3. Corollary 1.2 of [GR], that tunnel number one knots in $S^{3}$ are $n$ string prime for all $n$, does not hold for knots with tunnel number greater than or equal to two.

Proof. For each $n \geq 3$ choose a knot $K$ satisfying the conditions of Theorem 1.1 and so that g.c.d. $\left(a_{i, j}\right) \neq 1$. By the proof of Theorem 1.1 it is an $n$-string composite and by $[\mathrm{LM}]$ it has tunnel number $n-1$.

Remark 5.4. A. Thompson shows, in [Th], that either a knot in $S^{3}$ in thin position is in bridge position or the knot complement contains a planar meridional incompressible surface (for both definitions see [Th] or [HK]). Based on this result D. Heath and T. Kobayashi developed a method to search for thin positions for knots. Theorem 1.1 implies that one cannot use both results to conclude that a knot in $S^{3}$ in thin position is in bridge position for all knots $K$ which admit a $2 n$-plat, $n \geq 3$ projection with $m \geq 5,\left|a_{i, j}\right|>1$, for each $i$ and $j$.

\section{ACKNOWLEDGEMENTS}

We would like to thank Ying Qing Wu for this version of the proof of Lemma 2.6 and for referring us to his paper [Wu 2]. We also thank Cameron Gordon for conversations regarding this work. Both contributed to a shorter and more elegant proof of Lemma 3.4 which was presented in this paper.

\section{REFERENCES}

[BZ] G. Burde and H. Zieschang, Knots, De Gruyter Studies in Mathematics, 5, New York, 1985. MR 87b:57004

[CGLS] M. Culler, C. Gordon, J. Luecke, and P. Shalen, Dehn surgery on knots, Ann. of Math. 125 (1987), 237-300. MR 88a:57026

[CL] D. Cooper and D. Long, Derivative varieties and the pure braid group, Amer. J. Math. 115 (1993), 137-160. MR 94b:57003

[Fi] E. Finkelstein, Closed incompressible surfaces in closed braid complements, J. Knot Theory Ramifications 7 (1998), 335-379. CMP 98:13

[GL] C. McA. Gordon and J. Luecke, Reducible manifolds and Dehn surgery, Topology 35 (1996), 385-409. MR 97b:57013

[GR] C. McA. Gordon and A. Reid, Tangle decompositions of tunnel number one knots and links, J. Knots Theory Ramifications 4 (1995), 389-409. MR 96m:57016

[He] J. Hempel, 3-manifolds, Ann. of Math. Studies 86, Princeton University Press, Princeton, N.J., 1976. MR 54:3702

[JS] W. Jaco and P. Shalen, Seifert fibered spaces in 3-manifolds, Mem. Amer. Math. Soc. 21 (1979). MR 81c:57010

[HK] D. Heath and T. Kobayashi, A search method for a thin position of a link, preprint.

[LM] M. Lustig and Y. Moriah, Generalized Montesinos knots, tunnels and $\mathcal{N}$-torsion, Math. Ann. 295 (1993), 167-189. MR 94b:57011

[LP] M. T. Lozano and J. H. Przytycki, Incompressible surfaces in the exterior of a closed 3-braid, Math. Proc. Cambridge Philos. Soc. 98 (1985), 275-299. MR 87a:57013

[Ly] H. Lyon, Incompressible surfaces in knot spaces, Trans. Amer. Math. Soc. 157 (1971), 53-62. MR 43:1169

[Me] W. Mensco, Closed incompressible surfaces in alternating knot and link complements, Topology 23 (1984), 37-44. MR 86b:57004

[Oe] U. Oertel, Closed incompressible surfaces in complements of star links, Pacific J. Math. 111 (1984), 209-230. MR 85j:57008 
[Sh] H. Short, Some closed incompressible surfaces in knot complements which survive surgery, London Math. Soc. Lecture Notes Ser., 95 (1985), 179-194. MR 88d:57006

[Sw] G. A. Swarup, On incompressible surfaces in the complements of knots, J. Indian Math. Soc. 37 (1973), 9-24. MR 50:14757

[Th] A. Thompson, Thin position and bridge number for knots in the 3-sphere, Topology $\mathbf{3 6}$ (1997), 505-507. MR 97m:57013

[Wu 1] Y. Q. Wu, Incompressibility of surfaces in surgered 3-manifolds, Topology 31 (1992), 271-279. MR 94e:57027

[Wu 2] Y. Q. Wu, The classification of nonsimple algebraic tangles, Math. Ann. 304 (1996), 457-480. MR 97b:57010

Department of Mathematics, (CUNY) Hunter College, New York, New York 10021

E-mail address: efinkels@shiva.hunter.cuny.edu

Department of Mathematics, Technion, Haifa 32000, Israel

E-mail address: ymoriah@techunix.technion.ac.il 